\title{
Do avanafil and zaprinast exert positive effects on bone tissue via the nitric oxide/cyclic guanosine monophosphate/protein kinase-G signaling pathway in rats with ovariectomy-induced osteoporosis?
}

\author{
Zübeyir Huyut $^{\oplus 1}$, Nuri Bakan², and Serkan Y1ldırım³ \\ ${ }^{I}$ Department of Biochemistry, Medical Faculty, Van Yuzuncu Yll University, Tuşba/Van, Turkey, \\ ${ }^{2}$ Department of Biochemistry, Medical Faculty, Ataturk University, Yakutiye, Erzurum, Turkey, \\ ${ }^{3}$ Department of Pathology, Faculty of Veterinary, Ataturk University, Yakutiye, Erzurum, Turkey
}

\begin{abstract}
Phosphodiesterase-5 inhibitors (PDE-5Is) exert positive effects on bone healing and mineralization by activation the nitric oxide/cyclic guanosine monophosphate/protein kinase-G (NO/cGMP/PKG) signaling pathway. In this study, the effects of zaprinast and avanafil, two PDE-5Is, on the NO signaling pathway, estrogen levels, selected bone formation and destruction marker levels, wholebody bone mineral density (WB-BMD), right femur trabecular bone thickness (RF-TBT) and epiphyseal bone width, angiogenesis in the bone-marrow, and selected oxidative stress parameter levels were investigated in rats with ovariectomy-induced osteoporosis. Twenty four adult rats (8 months old) were equally divided into four groups. The first group was the sham operated group. Groups 2, 3 and 4 included ovariectomized rats. At six months after ovariectomy, the $3^{\text {rd }}$ and $4^{\text {th }}$ groups were administered $10 \mathrm{mg} / \mathrm{kg}$ zaprinast and avanafil daily as a single dose for 60 days, respectively. Increases in the activity of the NO/cGMP/PKG signalling-pathway, C-terminal collagen peptide levels, angiogenesis in the bone marrow, RF-TBT, epiphyseal bone width and WB-BMD were observed compared to the ovariectomized positive control group (OVX), while the pyridinoline and deoxypyridinoline levels were decreased in the OVX+zaprinast and OVX+avanafil groups $(\mathrm{p}<0.05)$. The malondialdehyde, ubiquinone10/ubiquinol10 and 8-hydroxy-2-deoxyguanosine $/ 10^{6}$ deoxyguanos ine levels were also increased in the ovariectomized groups compared to the sham group $(\mathrm{p}<0.05)$. Based on these results, the levels of bone atrophy and some markers of oxidative stress were increased due to acute estrogen deficiency induced by ovariectomy, but zaprinast and avanafil administration significantly prevented these changes.
\end{abstract}

Key-words: Bone mineral density. Osteoporosis. Ovariectomy. Oxidative stress. Phosphodiesterase-5 inhibitor.

\section{INTRODUCTION}

Osteoporosis (OP) is an important public health problem characterized by a deterioration of the bone tissue microstructure and diminished density, resulting in an increased risk of fragility (Wu et al., 2017). Osteoporosis is a disease that usually remains silent and is not noticed until a fracture occurs. This disease, which is painless in its initial stages, is accompanied by back pain, a shortening

*Correspondence: Z. Huyut. Department of Biochemistry. Faculty of Medicine. Yuzuncu Yil University. 65080-Van, Turkey, Phone: +90 432225 17 01-05. Fax: +90 432236 1054. e-mail: zubeyir.huyut@gmail.com of height, spinal deformation and an increasing incidence of fractures due to sudden collapse of the vertebrae in advanced cases (Kanis, Johnell, 2002).

More than 200 million people suffer from OP. The annual number of fractures is estimated to increase by $50 \%$ by 2025 , with an annual cost of $\$ 25$ billion (Zhou et al., 2017). Currently, pharmacological doses of estrogen, selective estrogen receptor modulators, hormone replacement therapy (HRP), biphosphonates and calcitonin are used to prevent or treat OP (El-Nabarawi, El-Wakd, Salem, 2017). However, alternative drugs and treatment modalities continue to be explored for the prevention or treatment of OP because of 
the adverse effects of hormone replacement therapy and the susceptibility of some patients to the medications used for treatment (Xing, Ni, Wang, 2017).

Recently, bone cells have recently been reported to secrete nitric oxide (NO) and nitric oxide synthase isoenzymes (NOSs) (Zhou et al., 2016; Mancini et al., 2000). The effect of NO on osteoblastic activity is not yet clear. Some authors reported that NO donors increase cGMP, levels and, alkaline phosphatase activity (ALP) and contribute to the formation of bone nodules, and osteocalcin release in vitro. Other authors reported an inhibitory effect of these compounds. Nevertheless, some physiological processes increase NO production and osteoblastic activity (Mancini et al., 2000). In addition, NO contributes to ameliorating bone damage and, has been shown to exert positive effects on osteoblasts, osteoclasts and osteocytes. NO, also exerts important regulatory effects on osteoblast and bone metabolism (Zhou et al., 2016).

In patients with OP, cyclic guanosine monophosphate (cGMP) mediates the intracellular effects of $\mathrm{NO}$ and through the activation of protein kinase- $\mathrm{G}$ (PKG) (Kass et al., 2007). The inhibition of the PDE-5 isoenzyme, which was hydrolyzes cGMP, by phosphodiesterase-5 inhibitors (PDE-5Is) such as sildenafil (Kaleta et al., 2017) effectively regulates the cardiovascular system and increase vascular tone by activating cGMP and PKG in the NO signaling pathway (Mancini et al., 2000). According to Yaman et al. (2011), PDE-5 inhibitors may effectively increase the vascular tone (angiogenesis) of bone tissue by activating cGMP, and use a similar pathway to regulate the NO signaling pathway in bone calcification and the transformation of osteoblast cells to osteoclasts. Furthermore, Yaman et al. (2011) showed that sildenafil a PDE-5 inhibitor, positively regulates to bone healing in rats with bone fractures. In addition, in studies by our group, the PDE-5 inhibitors vardenafil, tadalafil and udenafil increased angiogenesis in the bone tissue via the NO/cGMP/PKG signaling pathway and positively contributed to bone mineralization (Alp et al., 2017; Huyut et al., 2018). Therefore, we postulated that among the PDE5Is, zaprinast and avanafil may be positively modulate bone mineralization by increasing angiogenesis in the bone tissue via the NO/cGMP/PKG signaling pathway.

Based on accumulating evidence, OP and oxidative stress are positively correlated (Zheng et al., 2016). As shown in the study by Zheng et al. (2016), an increase in oxidative stress or decrease in the antioxidant capacity positively contributed to the development of OP. NO may cause oxidative stress by inducing the formation of some reactive oxygen species (ROS), such as peroxynitrite (ONOO-) radicals, which are also believed to exert some beneficial effects (Rahal et al., 2014). Therefore, researchers have asked how the activation of the NO metabolic pathway by avanafil and zaprinast will affect NO levels and oxidative stress in rats subjected to an ovariectomy.

In this study, the effects of zaprinast and avanafil on bone tissues and oxidative stress mediated by the NO signaling pathway in experimental animals with ovariectomy were analyzed. For this purpose, we investigated the biochemical effects of zaprinast and avanafil on the levels of endothelial nitric oxide synthase (NOS), NO, cGMP, PKG, PDE-5 (the factors associated with the NO signaling pathway), pyridinoline, deoxypyridinoline (the bone resorption indicators), C-terminal collagen peptide, procollagen peptide (bone formation markers), the lipid peroxidation end product malondialdehyde (MDA), $8-\mathrm{OHdG} / 10^{6} \mathrm{dG}$ (oxidative DNA damage indicator), and $\mathrm{CoQ} 10 / \mathrm{CoQ} 10 \mathrm{H}$ (oxidative mitochondrial damage indicator). The effects of zaprinast and avanafil on the RFTBT, epiphyseal bone width, and angiogenesis in the right femur bone marrow were also assessed.

\section{MATERIAL AND METHODS}

\section{Chemicals}

All chemicals and drugs were of analytical grade and purchased from commercial sources. Zaprinast, thiobarbituric acid, 1,1,3,3-tetraethoxypropane, CoQ10, 8 -OHdG, dG 10\% formalin and phosphate buffer were purchased from Sigma (Z0878, T5500, T9889, C9538, H5653, 854999, HT501128, P4417, respectively, Sigma Aldrich, USA). The osteosoft solution and avanafil were purchased from Merck (Merck, HC 313331, Germany) and PubChem (CID: 330784-47-9), respectively.

\section{Experimental Design and OP Development Process}

The Van Yuzuncu Y1l University Experimental Animals Local Ethics Committee Presidency approved this study. Twenty four female albino rats (8 months old) were randomly divided into four groups with equal numbers of animals $(n=6)$ : sham, ovariectomized (OVX), OVX + zaprinast and OVX + avanafil groups. The dose and time of inhibitors in the present study were determined according to dose and time used in previous studies for PDE-5Is (Yaman et al., 2011; Alp et al., 2017; Huyut et al., 2018). During the study, the 
Do avanafil and zaprinast exert positive effects on bone tissue via the nitric oxide/cyclic guanosine monophosphate/protein kinase-G signaling pathway in rats with ovariectomy-induced osteoporosis?

rats in all groups were provided standard pellet feed and water and housed on a $12 \mathrm{~h} \mathrm{light/dark} \mathrm{cycle.} \mathrm{The}$ procedure used to establish the osteoporosis in rats was described by Lien et al. (2009). For this purpose, after disinfecting the abdominal area of anesthetized rats (20 $\mathrm{mg} / \mathrm{kg}$ of Ketazol, Richte Pharma, Wels, Austria) with a $75 \%$ ethanol solution, an approximately $2 \mathrm{~cm}$ incision was made in the abdomen. After the overies were removed, the uterus and fat tissue were again placed in the abdominal cavity and the abdominal area was sutured. Post-operative care was administered to rats housed in separate cages. After postoperative care, OP was allowed to develop for six months. At the end of 6 months, whole body bone mineral density was measured using dual energy X-ray absorptiometry (DEXA) to determine whether osteoporosis developed. The following groups were established:

Group 1: Under anesthesia, the abdomen of each rat was surgically incised approximately $2 \mathrm{~cm}$ and closed again. After postoperative care, the animals were fed standard pellet feed until the end of the study (Sham group).

Group 2: Under anesthesia, the abdominal areas of the rats were incised approximately $2 \mathrm{~cm}$, the ovaries were removed, and the incisions were closed again. After postoperative care, the animals were fed standard pellet feed until the end of the study (positive control group or OVX).

Group 3: Under anesthesia, the abdominal areas of the rats were surgically incised approximately $2 \mathrm{~cm}$, the ovaries were removed, and the incisions were closed again. After postoperative care, the animals were fed standard pellet feed for six months. At the end of six months, $10 \mathrm{mg} / \mathrm{kg}$ zaprinast was also administered orally as a single daily dose as along with standard pellet feed for two months. (OVX+zaprinast).

Group 4: Under anesthesia, the abdominal areas of the rats were surgically incised approximately $2 \mathrm{~cm}$, the ovaries were removed, and the incisions were closed again. After postoperative care, the animals were fed standard pellet feed for six months. At the end of six months, $10 \mathrm{mg} / \mathrm{kg}$ avanafil was also administered orally as a single daily dose as along with standard pellet feed for two months (OVX+avanafil).

\section{Radiological Measurement of WB-BMD}

Whole body bone mineral density (WB-BMD) was measured by scanning the whole bodies of anesthetized rats in all groups with a dual-energy $\mathrm{X}$ ray absorptiometry (DEXA) device (Hologic, QDR-Discovery C Hologic, Inc., Waltham, MA), before and after the ovariectomy and inhibitor administration three separate times. The results are presented as $\mathrm{g} / \mathrm{cm}^{2}$.

\section{Collection of Urine Samples}

Before and after the ovariectomy and administration of inhibitor therapy, 24-hour urine samples were collected from all rats in metabolic cages and stored at $-80{ }^{\circ} \mathrm{C}$ until analyses. The levels of pyridinoline (PD) and deoxypyridinoline (DPD), bone resorption markers, were measured in urine samples.

\section{Collection of Blood and Bone Tissue Samples}

After treatment, rats were anesthetized and blood samples were collected via an intracardial puncture. Some of the whole blood that was collected in the hemogram tubes with the help of the injector was centrifuged at $2500 \mathrm{xg}$ for $15 \mathrm{~min}$ and the resulting plasma samples were divided into aliquots and stored at $-80{ }^{\circ} \mathrm{C}$ until further analysis. The other part of the whole blood was reserved for 8-OHdG and dG measurements. In addition, the right femur tissues of rats were preserved in $10 \%$ formalin for histopathological and immunological examinations.

\section{Biochemical Measurements}

The plasma levels of eNOS, NO PDE-5, cGMP and $\mathrm{PKG}$, which are associated with the NO signaling pathway, $\mathrm{C}$-terminal collagen peptide (CTCP) and procollagen peptide (PICP), which indicate bone activity, urine levels of pyridinoline (PD) and deoxypyridinoline (DPD), which are known as bone destruction markers in urine, and plasma estrogen levels were measured with commercially available ELISA kits according to the manufacturer's instructions (Eastbiopharm).

\section{Measurement of MDA Levels}

Plasma MDA levels were analyzed using the method reported by Khoschsorur et al. (2000). We added the following solutions to each $50 \mu \mathrm{L}$ plasma sample: 750 $\mu \mathrm{L}$ of $0.44 \mathrm{M} \mathrm{H}_{3} \mathrm{PO}_{4}, 250 \mu \mathrm{L}$ of $0.25 \mathrm{mM}$ thiobarbituric acid (TBA) and $450 \mu \mathrm{L}$ of distilled water. The mouths of the tubes were tightly closed and then the tubes were placed in a boiling water bath for $60 \mathrm{~min}$. Next, the tubes 
were cooled with tap water. An alkaline methanol solution ( $50 \mathrm{~mL}$ of methanol+4.5 $\mathrm{mL}$ of $1 \mathrm{M} \mathrm{NaOH}$ ) was added to the solution in each tube at a $1: 1(\mathrm{v} / \mathrm{v})$ ratio. The mixture was centrifuged at $2500 \mathrm{xg}$ for $3 \mathrm{~min}$. Two hundered microliters of the supernatant in the upper phase was removed and placed in a vial. Then, it was transferred to the high pressure liquid chromatography (HPLC) device. An RP18 column (length of $150 \times 4.6 \mathrm{~mm}$ and $5 \mu \mathrm{m}$ particle size) was used for separation. The mobile phase was a mixture of $400 \mathrm{~mL}$ of $50 \mathrm{mM}$ phosphate buffer ( $\mathrm{pH}: 6.8$ ) and $600 \mathrm{~mL}$ of pure methanol. The flow rate of the device was set to $0.8 \mathrm{~mL} / \mathrm{min}$ and the injection volume to 20 $\mu \mathrm{L}$. The concentration of the MDA-TBA complex was measured and compared with standard samples of 1,1,3,3 tetraethoxypropane prepared at different concentrations in the fluorescence detector at an excitation wavelength of $527 \mathrm{~nm}$ and and emission wavelength of $551 \mathrm{~nm}$. The obtained results are reported in $\mu \mathrm{M}$.

\section{Measurement of CoQ10 Levels}

Plasma CoQ10 levels were measured using the method reported by Littarru et al. (2004).

Oxidized CoQ10: Fifty microliters of benzoquinone ( $2 \mathrm{mg} / \mathrm{mL}$ ) were added to $200 \mu \mathrm{L}$ of the plasma sample and vortexed for $10 \mathrm{sec}$. After an incubation at room temperature for $10 \mathrm{~min}, 1 \mathrm{~mL}$ of propanol was added. The mixture was vortexed for $10 \mathrm{sec}$ and centrifuged at $4000 \mathrm{xg}$ for $6 \mathrm{~min}$. Two hundered microliters of the supernatant from the upper phase was placed in a vial and loaded onto the HPLC apparatus. A mixture of 650 $\mathrm{mL}$ of ethanol and $350 \mathrm{~mL}$ of methanol was used as the mobile phase. A C18 column with a $5 \mu \mathrm{m}$ particle size and a length of $25 \times 4.6 \mathrm{~mm}$ was used for separation. The flow rate of the device was set to $0.8 \mathrm{~mL} / \mathrm{min}$ and the injection volume was set to $20 \mu \mathrm{L}$. The concentration was measured and compared with standards prepared at different concentrations in the UV detector at $275 \mathrm{~nm}$.

Total CoQ10; Fifty microliters of benzoquinone ( $2 \mathrm{mg} /$ $\mathrm{mL}$ ) were added to $200 \mu \mathrm{L}$ of the plasma sample and vortexed for $10 \mathrm{sec}$. Then, $1 \mathrm{~mL}$ of lithium perchlorate was added to the mixture. After an incubation at room temperature for $10 \mathrm{~min}, 1 \mathrm{~mL}$ of propanol was added to the solution. The mixture was vortexed for $10 \mathrm{sec}$ and centrifuged at $4000 \mathrm{xg}$ for $6 \mathrm{~min}$. Two hundered microliters of the supernatant were placed in a vial and loaded onto the HPLC device. A mixture of pure ethanol-pure methanol and lithium perchlorate $(50 \mathrm{mM})$ at a volumetric ratio of 70:20:10 was used as the mobile phase. A C18 column with a length of $25 \times 4.6$ and a $5 \mu \mathrm{m}$ particle size was used for separation. The flow rate of the device was set to $0.8 \mathrm{~mL} / \mathrm{min}$ and the injection volume to $20 \mu \mathrm{L}$. Measurements were performed and compared with the standards prepared at different concentrations in the UV detector at $275 \mathrm{~nm}$. The results are reported as the CoQ10/CoQ10H ratio using the CoQ10H: CoQ10/ Total CoQ10 - oxide CoQ10 equation.

\section{Measurement of 8-OHdG and dG Levels}

Leukocyte DNA was isolated from whole blood samples using a DNA isolation kit (Invitrogen, CA, USA) according to the manufacturer's instructions. As described by Kaur and Halliwell (1996), the 150 $\mu \mathrm{L}$ DNA sample was added to the same volume of pure formic acid and incubated at $150{ }^{\circ} \mathrm{C}$ for $30 \mathrm{~min}$. Then, $100 \mu \mathrm{L}$ of acetonitrile were added. The levels of 8-OHdG were measured at $600 \mathrm{mV}$ in the ECD and the levels of dG were measured at $275 \mathrm{~nm}$ in the UV detector and compared with different standards using HPLC. The mobile phase was prepared by mixing 30 $\mathrm{mL}$ of acetonitrile with $970 \mathrm{~mL}$ of phosphate buffer $(\mathrm{pH}$ 5.5 and $50 \mathrm{mM}$ ). A C18 column with a length of $15 \times 4.6$ $\mathrm{mm}$ and a $5 \mu \mathrm{m}$ particle size was used. The flow rate of the device was set to $0.8 \mathrm{~mL} / \mathrm{min}$ and the injection volume to $20 \mu \mathrm{L}$. The results are reported as the number of $8-\mathrm{OHdG} / 10^{6} \mathrm{dG}$.

\section{Histopathological and Immunohistochemical Evaluation of Right Femur Bone Tissue}

At the end of the necropsy, the right femur tissues used for the histopathological evaluation were fixed with a $10 \%$ formalin solution for $48 \mathrm{~h}$. Bone tissues were incubated in osteosoft solution (MERC, HC313331, Germany) for 96-120 h to decalcify the tissues. Then, the tissues were washed with tap water for $24 \mathrm{~h}$. After routine processes used for tissue tracking, samples were embedded in paraffin blocks. Sections were cut from each block at $4 \mu \mathrm{m}$ thickness and placed on the slides. Sections were stained with hematoxylin-eosin (HE) for the histopathological examination and the right femur epiphyseal bone width and mineral density were visualized under a light microscope (Leica DM1000).

Immunohistochemical staining was performed using the method described by Shi et al. (1996). After 
Do avanafil and zaprinast exert positive effects on bone tissue via the nitric oxide/cyclic guanosine monophosphate/protein kinase-G signaling pathway in rats with ovariectomy-induced osteoporosis?

deparaffinization and dehydration, samples were heated 4 times in antigen retrieval solution (citrate buffer, $\mathrm{pH}$ 6.1) in a microwave for $5 \mathrm{~min}$ each. After the preparations were cooled and washed, they were dried and a line was drawn around the sections with a special glass pen. Endogenous peroxidase activity was inhibited by washing the sections with phosphate buffered saline (PBS, $\mathrm{pH}$ 7.2) for $5 \mathrm{~min}$ and incubating them with a $3 \% \mathrm{H}_{2} \mathrm{O}_{2}$ solution for $10 \mathrm{~min}$. After washes with PBS, the sections were incubated with a protein block compatible with all primary and secondary antibodies to prevent nonspecific background staining for $5 \mathrm{~min}$. Then, sections were incubated with a primary antibody against CD-31 (PA5-16301; Thermo Scientific, USA) at room temperature for 1 hour. Sections were washed with PBS for $10 \mathrm{~min}$ and then incubated with the specific antipolyvalent HRP detection IHC kit (CAT: TP-060-HL, Thermo Scientific, USA) at room temperature for 10-30 min according to the manufacturer's recommendations. After repeated washes, 3-amino-9-ethyl-carbazole (AEC) was added to the sections as a chromogen and incubated for 5-10 min. The sections were incubated with Mayer's hematoxylin for 1-2 minutes for background staining and washed with tap water. Sections were covered with coverslips using water-based glue and angiogenesis in the right femur bone marrow was examined under a light microscope (Leica DM1000).

\section{Statistical Analyses}

Descriptive statistics are reported as the means and standard deviations. Following ANOVA, Duncan's multiple comparison test was used to identify differences between groups. Pearson's correlation coefficients were calculated separately for the each group to determine the relationship between the variables after the OVX and inhibitor administration. The statistical significance level in the calculations was set to $5 \%(\mathrm{p}<0.05)$ and the SPSS statistical package program (version 15) was used for the calculations.

\section{RESULTS AND DISCUSSION}

We measured the weights of animals in the all groups before the ovariectomy and inhibitor administration. The average weights of the rats were $285 \pm 17,288 \pm 14,292 \pm 18$ and $282 \pm 11 \mathrm{~g}$ in the sham, OVX, OVX+zaprinast and OVX+avanafil groups, respectively, and the difference between groups was not siginificant. After the OVX and the administration of the inhibitors, the average weights were $321 \pm 13$, $314 \pm 16,316 \pm 12$ and $319 \pm 15 \mathrm{~g}$ in the control, OVX, OVX+zaprinast and OVX+avanafil groups, respectively. A significant difference was not observed between of groups after OVX and inhibitor application. In addition, a partial change in weights was observed before and after OVX and inhibitor application between the same groups due to the approximately eight month process of the OVX and inhibitor administration.

\section{Changes in Estrogen Levels Before and After Ovariectomy}

Estrogen levels were dramatically decreased in ovariectomized groups (approximately 44\%). However, the greatest decrease was observed in the OVX+zaprinast group ( $\mathrm{p}<0.05$, Table I).

The high prevalence of fractures and osteoporosis in women is associated with postmenopausal bone loss due to an estrogen deficiency in the postmenopausal stage (Mada et al., 2017). For this reason, we used the common practice of ovariectomy to induce OP in experimental animals and produce an acute estrogen deficiency (Sharad et al., 2017). In a previous study, rats with ovariectomy presented dramatically decreased estrogen levels (approximately $45 \%$ ) compared with the sham group (Chow et al. 2014). In the present study, estrogen levels in the ovariectomized groups were approximately $49.44 \%$ lower than those in the sham group $(\mathrm{p}<0.05)$. Based on these results, an acute estrogen deficiency, which was the goal of the ovariectomy, was succesfully established. 
TABLE I - Comparison of the plasma levels of intermediates in the NO signaling pathway, selected bone formation and degradation markers, cortisol and selected oxidative stress parameter values in rats with ovariectomy-induced osteoporosis

\begin{tabular}{lcccc}
\hline & Control & OVX & OVX + Zaprinast & OVX + Avanafil \\
\hline eNOS $(\mathrm{ng} / \mathrm{mL})$ & $48.888 \pm 0.428$ & $51.035 \pm 0.374^{*}$ & $53.509 \pm 0.291^{\ddagger}$ & $56.466 \pm 0.753^{\varphi}$ \\
\hline NO $(\mu \mathrm{mol} / \mathrm{L})$ & $155.659 \pm 2.191$ & $183.269 \pm 3.091^{*}$ & $167.591 \pm 2.829^{\ddagger}$ & $194.705 \pm 3.640^{\varphi}$ \\
\hline PDE-5 $(\mathrm{ng} / \mathrm{mL})$ & $4.411 \pm 0.190$ & $4.335 \pm 0.220$ & $3.988 \pm 0.266^{\ddagger}$ & $3.860 \pm 0.250^{\ddagger}$ \\
\hline cGMP $(\mathrm{pmol} / \mathrm{L})$ & $42.968 \pm 0.606$ & $47.305 \pm 0.445^{*}$ & $48.608 \pm 0.422^{\ddagger}$ & $50.343 \pm 0.619^{\varphi}$ \\
\hline PKG $(\mathrm{ng} / \mathrm{mL})$ & $11.669 \pm 0.126$ & $12.041 \pm 0.229^{*}$ & $12.037 \pm 0.122^{*}$ & $14.371 \pm 0.246^{\varphi}$ \\
\hline PICP $(\mathrm{ng} / \mathrm{mL})$ & $62.291 \pm 0.637$ & $72.141 \pm 0.299^{\varphi}$ & $70.595 \pm 0.666^{*}$ & $70.413 \pm 0.893 *$ \\
\hline CTCP $(\mathrm{ng} / \mathrm{mL})$ & $5.696 \pm 0.085$ & $5.679 \pm 0.092$ & $5.809 \pm 0.061^{\neq}$ & $6.315 \pm 0.098^{\varphi}$ \\
\hline Estrogen $(\mathrm{ng} / \mathrm{L})$ & $36.335 \pm 2.411$ & $20.914 \pm 2.432^{*}$ & $14.384 \pm 1.867^{\varphi}$ & $19.839 \pm 0.912^{*}$ \\
\hline MDA $(\mu \mathrm{M})$ & $1.933 \pm 0.144$ & $1.992 \pm 0.090$ & $1.883 \pm 0.099$ & $1.918 \pm 0.148$ \\
\hline CoQ10/CoQ10H & $0.271 \pm 0.033$ & $0.279 \pm 0.059$ & $0.268 \pm 0.031$ & $0.265 \pm 0.027$ \\
\hline 8-OHdG/10 dG & $0.721 \pm 0.067$ & $0.774 \pm 0.086$ & $0.732 \pm 0.081$ & $0.684 \pm 0.069$ \\
\hline
\end{tabular}

"* $\mathrm{p}$ ": Compared to the control group in each line $(\mathrm{p}<0.05)$. " $\mathrm{p}$ ": Compared to the control and OVX groups in each line $(p<0.05)$. "“p": Compared to the other groups in each line $(p<0.05)$. eNOS: endothelial nitric oxide synthase, NO: nitric oxide, PDE-5: phosphodiesterase-5, cGMP: cyclic guanosine mono phosphate, PKG: protein kinase G, PICP: pro-collagen peptide, CTCP: c-terminal collagen peptide, MDA: malondialdehyde, CoQ10: ubiquinone 10, 8-OHdG: 8-hydroxy-2-deoxyguanosine, dG: deoxyguanosine.

\section{Changes in Parameters Related to the NO Signaling Pathway}

The levels of eNOS and NO, which are associated with the NO signaling pathway, were increased in the OVX group compared to the sham group $(p<0.05)$, and a significant change in cGMP and PKG levels was not observed between the same groups. However, a greater increase in the eNOS, cGMP and PKG levels was observed in the OVX+zaprinast and particularly the OVX+avanafil groups than in the OVX group $(\mathrm{p}<0.05)$. In addition, significantly lower PDE-5 levels were observed in the OVX+zaprinast and OVX+avanafil groups than in the sham and OVX groups $(\mathrm{p}<0.05$, Table I).

Many factors prevent the development of OP. One of these possible factors is eNO, which is synthesized by the
NOS enzyme and inhibits osteoclastic bone resorption in vitro and regulates bone regeneration in vivo (Fan et al., 2004). According to one study, eNOS may spesifically mediate effects of estrogen on osteoblasts (Firat et al., 2009). As shown in the study by Armour et al. (2001) the eNOS signaling pathway plays an important role in regulating bone turnover and density by regulating osteoblastic function (Armour et al., 2001). Furthermore, Wimalawansa et al. showed that NO reduces bone loss in humans (Wimalawansa et al., 2000). NO exerts this positive effect on bone tissue through cGMP and PKG (Anderson et al., 2001). The continuation of the effect of NO depends on the persistent activity of cGMP. However, cGMP is hydrolyzed by the PDE-5 isoenzyme (Wie et al,. 2017). Thus, the inhibition of PDE-5 by PDE-5Is may exert a positive effect on bone tissue by 
Do avanafil and zaprinast exert positive effects on bone tissue via the nitric oxide/cyclic guanosine monophosphate/protein kinase-G signaling pathway in rats with ovariectomy-induced osteoporosis?

accelerating angiogenesis and blood flow in the bone tissue through a mechanism depending on the increased activity of cGMP (Yaman et al., 2011; Alp et al., 2017). Yaman et al. reported a positive contribution of the PDE-5 inhibitors sildenafil on reversing bone damage (Yaman et al., 2011). In previous studies by our group, PDE-5 inhibitors such as vardenafil, tadalafil, udenafil, avanafil and zaprinast enhanced angiogenesis in bone tissue and positively contributed to bone mineralization by maintaining the activity of the NO signaling pathway. Vardenafil, tadalafil and udenafil increase cGMP and PKG levels and decrease PDE-5 levels in female rats with ovariectomy induced OP (Alp et al., 2017; Huyut et al., 2018). In the present study, the PDE-5 inhibitors zaprinast and avanafil were used because their effects on the NO signaling pathway and bone tissue in rats with osteoporosis induced by ovariectomy have not been investigated. The data from the present study are also consistent with a previous study performed by our group (Alp et al., 2017). In addition, the levels of PDE-5, cGMP and PKG in the OVX+zaprinast and $\mathrm{OVX}+$ avanafil groups indicate that the $\mathrm{NO} / \mathrm{cGMP} / \mathrm{PKG}$ signaling pathway is activated and PDE-5 is effectively inhibited by zaprinast and avanafil.

\section{Changes in WB-BMD Before and After Ovariectomy}

WB-BMD values recorded before the ovariectomy operation were $0.188 \pm 0.013,0.186 \pm 0.003,0.190 \pm 0.006$, and $0.187 \pm 0.006 \mathrm{~g} / \mathrm{cm}^{2}$ in the sham, OVX, OVX+zaprinast and OVX+avanafil groups, respectively, and the difference between groups was not significant them $(p>0.05)$. Six months after the ovariectomy operation, we measured WB-BMD values to monitor the occurrence of osteoporosis and observed values of $0.209 \pm 0.005$, $0.182 \pm 0.004,0.185 \pm 0.006$, and $0.183 \pm 0.006 \mathrm{~g} / \mathrm{cm}^{2}$ in the sham, OVX, OVX+zaprinast and OVX+avanafil groups, respectively. Significantly lower levels were observed in the groups subjected to ovariectomy than in the sham group $(\mathrm{p}<0.05)$. After treatment with zaprinast and avanafil, WB-BMD values were $0.208 \pm 0.001$, $0.183 \pm 0.006,0.205 \pm 0.002$, and $0.218 \pm 0.004 \mathrm{~g} / \mathrm{cm}^{2}$ in the sham, OVX, OVX+zaprinast and OVX+avanafil groups, respectively. The BMD values of the OVX+zaprinast and OVX+avanafil groups were significantly higher than the OVX group $(p<0.05)$. In addition, BMD values of the OVX+zaprinast and OVX+avanafil groups were significantly higher than the values recorded before the inhibitor treatment $(\mathrm{p}<0.05$, Figure 1$)$.

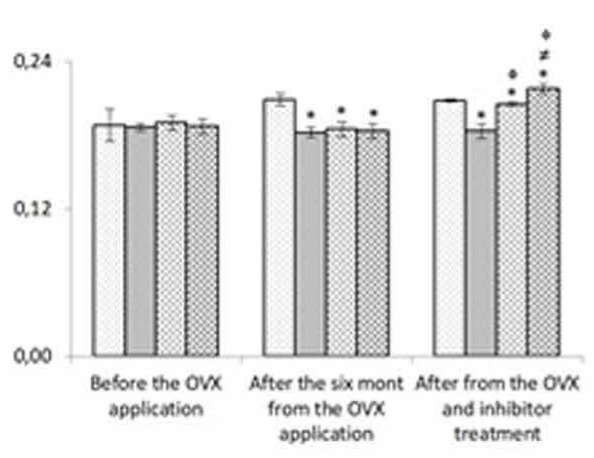

(A)

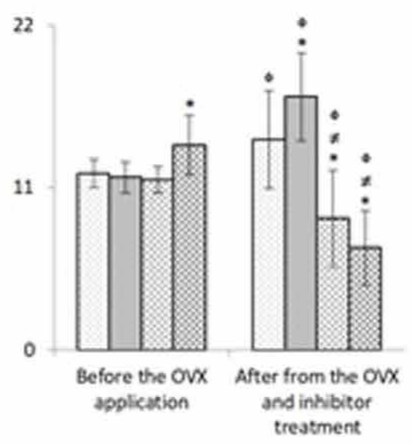

(B)

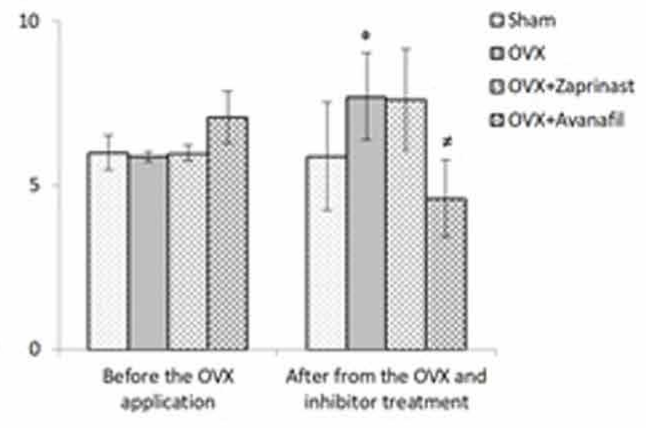

(C)

FIGURE 1 - Comparison of bone mineral density and urine pyridinoline and deoxypyridinoline levels before and after the OVX and inhibitor application. (A): Radiological measurement of bone mineral density $\left(\mathrm{g} / \mathrm{cm}^{2}\right)$. (B): Urine pyridinoline levels (nmol/ $\mathrm{mL}$ ). (C): Urine deoxypyridinoline levels $(\mathrm{nmol} / \mathrm{mL}) .{ }^{*} \mathrm{p}<0.05$ : compared to the control gorup, ${ }^{\neq} \mathrm{p}<0.05$ : According to the positive control group (OVX). ${ }^{\circ} \mathrm{p}<0.05$ : compared with the values recorded before the inhibitor treatment in the same group.

BMD is one of the most important parameters used to asses bone quality and diagnose OP (Martineau, Leslie, 2017). According to Li et al. (2014), the BMD is decreased in ovariectomized rats compared with the sham group, but the authors administered different doses of naringin significantly restricted the reduction of BMD. In a study conducted by our group, PDE-5 inhibitors such as vardenafil and tadalafil positively modulated the BMD 
(Alp et al., 2017; Huyut et al., 2018). In the literature review, we did not identify any studies investigating the effects of zaprinast and avanafil on BMD in osteoporotic animal or human models. The present study provides important new information about the effects of these drugs on BMD for the field. The lower BMD values of the positive control groups with ovariectomy compared to the sham group indicated that OP was established in the ovariectomized rats. Furthermore, the increasing BMD values observed in groups with an ovariectomy that were treated with zaprinast and avanafil compared to the ovariectomized positive control group showed that zaprinast and avanafil exerted positive effects on WBBMD. In addition, the observation of a more pronounced positive effect on the avanafil group indicated that avanafil prevented BMD loss due to acute estrogen deficiency better than zaprinast.

\section{Changes in Markers of Bone Formation and Destruction}

Urine levels of PD and DPD are used as bone destruction markers. In a previous study by our group, vardenafil, tadalafil and udenafil increased CTCP and PICP levels and reduced PD and DPD levels compared to the OVX group (Alp et al., 2017). Similar results were also obtained in this study.

Before the ovariectomy and inhibitor treatment, PD levels in the OVX, OVX+zaprinast and OVX+avanafil groups were $11.933 \pm 0.964,11.677 \pm 1.057,11.548 \pm 0.893$, and $13.890 \pm 1.995 \mathrm{nmol} / \mathrm{mL}$, respectively, and the difference between groups was not significant $(\mathrm{p}>0.05)$. Only the PD levels in the OVX+avanafil group were higher than those in the other groups $(\mathrm{p}<0.05)$. After ovariectomy and inhibitor treatment, the PD levels in the OVX, OVX+zaprinast and OVX+avanafil groups were $14.254 \pm 3.341,17.146 \pm 2.989$, $8.887 \pm 3.278$, and $6.906 \pm 2.516 \mathrm{nmol} / \mathrm{mL}$, respectively, and the PD level in the OVX group was higher than in the other groups $(p<0.05)$. Significantly lower PD levels were observed in the OVX+zaprinast group and particularly the OVX+avanafil group than in both the sham and OVX groups (Figure 1, $\mathrm{p}<0.05$ ). The DPD levels also displayed similar trends. Before ovariectomy and inhibitor treatment, the DPD levels in the sham, OVX, OVX+zaprinast and OVX+avanafil groups were $51.688 \pm 5.489,52.920 \pm 2.440$, $51.201 \pm 3.796$ and $54.962 \pm 4.721 \mathrm{nmol} / \mathrm{mL}$, respectively, and the difference between groups was not significant ( $p>0.05$ ). After ovariectomy and inhibitor treatment, the DPD levels in the sham, OVX, OVX+zaprinast and OVX+avanafil groups were $59.819 \pm 6.737,70.321 \pm 8.360,61.741 \pm 7.473$ and $51.477 \pm 8.212 \mathrm{nmol} / \mathrm{mL}$, respectively, and levels in the OVX+avanafil group were lower than the OVX group $(p<0.05$, Figure 1$)$. In addition, after ovariectomy and inhibitor treatment, DPD levels in the OVX group were significantly increased $(\mathrm{p}<0.05)$ compared with levels measured before ovariectomy and inhibitor treatment, but the differences between the other groups were not significant ( $\mathrm{p}>0.05)$.

In the present study, plasma CTCP and PICP levels and urine DPD and PD levels were also investigated. Osteocalcin, collagen peptide-I (CTX), PICP and homocysteine are known to be the most important serum markers of bone formation and turnover, reflecting osteoblastic activity (Zhang et al., 2015). When the levels of the bone formation markers CTCP and PICP were examined (Table I), we detected higher PICP levels in the OVX, OVX+zaprinast, and OVX+avanafil groups than in the sham group $(p<0.05)$. Higher PICP levels were observed in the OVX group than in the OVX+zaprinast and OVX+avanafil groups ( $\mathrm{p}<0.05$ ). In addition, the CTCP levels were observed in the OVX+zaprinast group and particularly the OVX+avanafil group than in the sham and OVX groups $(\mathrm{p}<0.05$, Table I).

Higher CTCP levels were observed in the $\mathrm{OVX}+$ zaprinast and OVX+avanafil groups than in the sham and OVX groups. In addition, observation that the avanafil group exhibited the highest levels revealed greater levels of osteoblastic activity and bone turnover in the avanafil group, consistent with the levels of other measured parameters. Higher PICP levels were observed in the ovariectomized groups. However, the observation of lower PICP levels in the OVX+zaprinast and OVX+avanafil groups than in the OVX group revealed an interesting situation that we did not expect. When PD, DPD and CTCP levels were evaluated in combination PD and DPD showed a significant negative correlation with CTCP levels and lower levels of bone destruction were observed, particularly in avanafil treated group.

\section{Immunohistochemical Staining and Histopathological Assesment of Changes in Right Femur Trabecular Bone Thicknes, Epiphyseal Bone Width, and Angiogenesis in Right Femur Bone Marrow}

A angiogenesis was significantly increased in the right femur bone tissues of the rats in the groups that underwent an ovariectomy compared to the sham group. 
Do avanafil and zaprinast exert positive effects on bone tissue via the nitric oxide/cyclic guanosine monophosphate/protein kinase-G signaling pathway in rats with ovariectomy-induced osteoporosis?

However, this increase was more pronounced in the OVX+zaprinast group and particularly OVX+avanafil group (Figure 2, Table II). The activation of the NO signaling pathway by zaprinast and particularly avanafil significantly increased angiogenesis in the right femur bone tissue.

PDE-5Is such as sildenafil, avanafil and zaprinast increase vasodilatation and blood flow by increasing the effect of cGMP (Kim et al,. 2004). In our previous study, the administration of vardenafil, tadalafil and udenafil to ovariectomized rats increased angiogenesis in bone tissue, as assessed by performing immunostaining with CD31 antibodies (Alp et al., 2017). CD31 has also been used to determine angiogenesis along with other antibodies. CD31 is normally expressed in endothelial cells, platelets, macrophages, Kupffer cells, granulocytes, lymphocytes, megakaryocytes, osteoclasts and neutrophils. CD31 is commonly used to visualize the presence of endothelial cells in histological sections. It is also used to show the degree of angiogenesis that reflects the growth rate in tumors and detect vein tumors, such as hemangioendotheliomas. In our study, the CD31 antibody was used for immunohistochemistry to examine angiogenesis in the right femur bone tissue (Miettinen, Lindenmayer, Chanbal, 1994). The findings were consistent with the important conclusions reported by previous authors about that PDE-5Is may positively contibute to better nutrition of the bone tissue and bone turnover by inducing angiogenesis in bone tissues of patients with osteoporosis.

The vertebral bones predominantly consist of the trabecular bone (trabecular bone 95\% and cortical bone 5\%). Therefore, a measurement of trabecular bone thickness in OP studies is important. Li et al.
(2014) reported an increased risk of fragility caused by a decrease in trabecular bone mineral density in the femur and femur head in the ovariectomized rats, and the administration of naringin significantly decreased the fragility. In the present study, we examined the right femur trabecular bone thickness histopathologically and observed decreased levels in the groups with ovariectomy compared to the sham group. However, this decrease was less obvious in the OVX+zaprinast group and particularly the OVX+avanafil group; similar results were observed compared to images from the control group (Figure 3). Histopathological images of the epiphyseal bone width also showed similar trends to images of trabecular bone density. The right femur epiphyseal bone width values of the all groups with ovariectomy were lower than the sham group $(p<0.05)$. However, this decrease was less evident in the OVX+zaprinast and OVX+avanafil groups than in the OVX group and was closer to the values of the control group (Figure 4 and Table II).

According to the data from the present study, the right femur trabecular bone thickness and epiphyseal bone width were significantly reduced in the positive control group with ovariectomy compared to the sham group. However, the right femur trabecular bone density and epiphyseal bone width were significantly preserved in the zaprinast treated ovariectomized rats compared to the positive control group with ovariectomy. In addition, the right femur trabecular bone density and epiphyseal bone width of the avanafil treated group with ovariectomy were similar to the sham group, and no animals in this group developed atrophy. Thus, zaprinast and avanafil had significant positive contributions to bone mineralization in ovariectomized female rats and significantly inhibited bone atrophy.

TABLE II - Comparison of right femur trabecular bone density, formation of new vascular structures and epiphyseal bone width in the all groups of rats with ovariectomy-induced osteoporosis

\begin{tabular}{lccr} 
Groups & $\begin{array}{c}\text { Right Femur Visual } \\
\text { Trabecular Density }\left(\mathbf{m m}^{2}\right)\end{array}$ & $\begin{array}{c}\text { Right Femur Visual New } \\
\text { Vessel Density }\left(\mathbf{m m}^{\mathbf{2}}\right)\end{array}$ & $\begin{array}{c}\text { Right Femur Epiphys } \\
\text { Width (px) }\end{array}$ \\
\hline Control & ++++ & + & $1487.032 \pm 54.689^{*}$ \\
OVX & + & ++ & $1094.853 \pm 43.030^{*}$ \\
OVX+Zaprinast & +++ & +++ & $1122.032 \pm 45.017^{*}$ \\
OVX+Avanafil & ++++ & ++++ & $1350.100 \pm 50.012^{*}$
\end{tabular}

"+": A semicantitavie visual evaluation of the results. *p<0.001: compared to the other groups. 

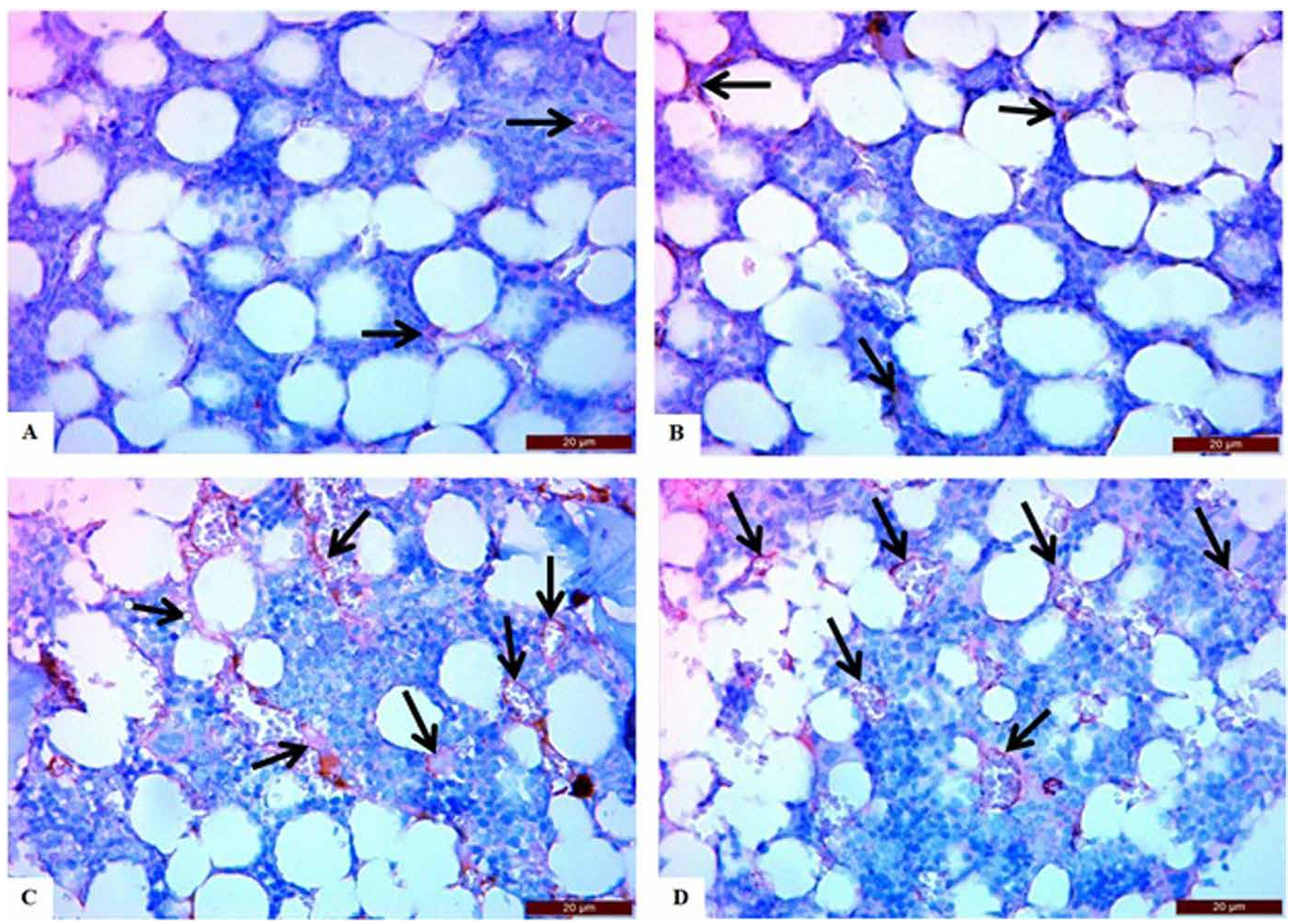

FIGURE 2 - Images showing vessel formation (angiogenesis) in the right femur bone marrow of rats with ovariectomy-induced osteoporosis. A: Control group, B: OVX group, C: OVX+zaprinast group and D: OVX+avanafil group. Red-pink staining shows the formation of new vascular structures in the sections. Scale bar: $20 \mu \mathrm{m}$.
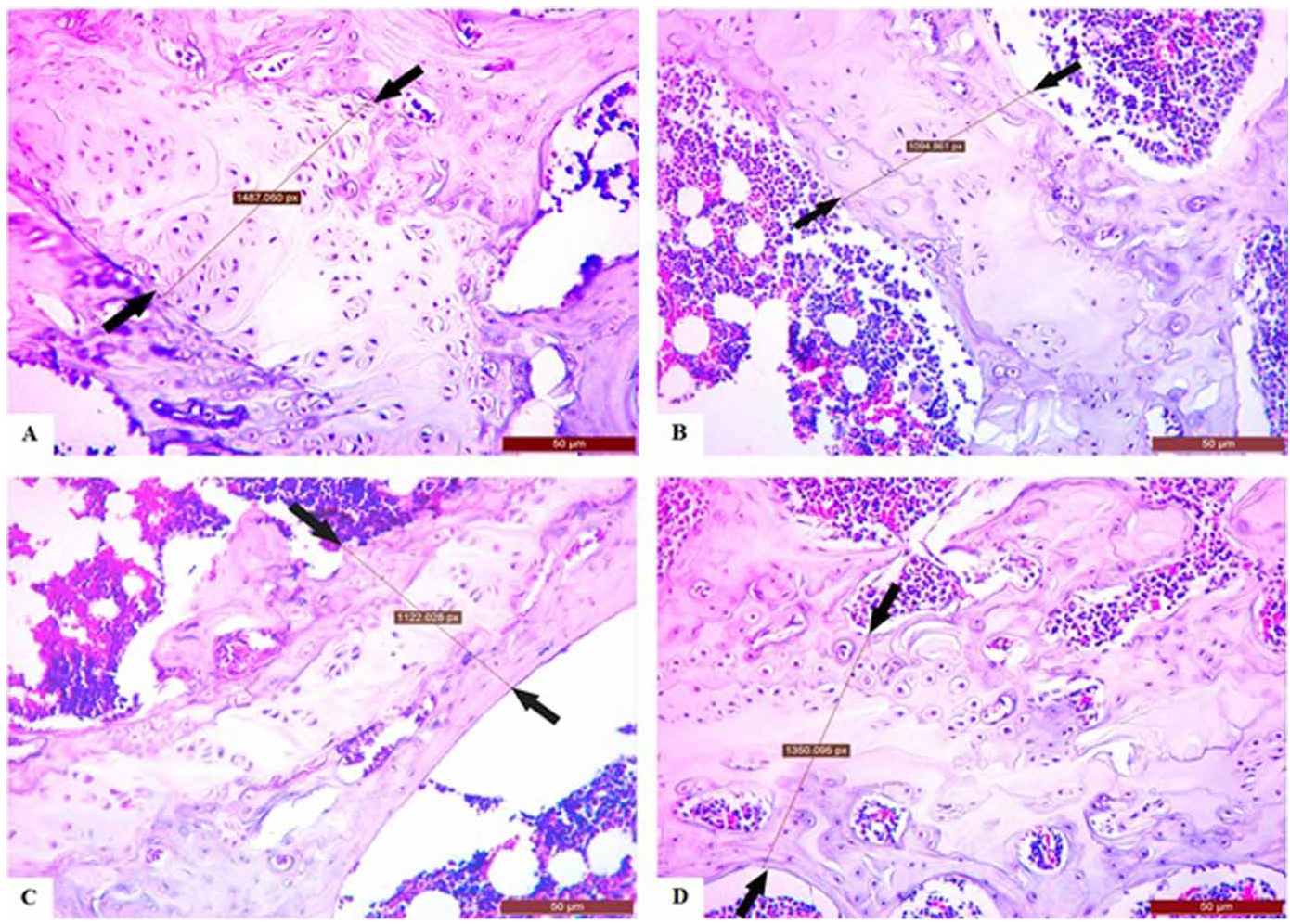

FIGURE 3 - Images showing the trabecular bone thickness of the right femur head in rats with ovariectomy-induced osteoporosis. A: Control group, B: OVX group, C: OVX+zaprinast group and D: OVX+avanafil group. The plain range or channels indicate mineral deposits. Scale bar: $100 \mu \mathrm{m}$. 
Do avanafil and zaprinast exert positive effects on bone tissue via the nitric oxide/cyclic guanosine monophosphate/protein kinase-G signaling pathway in rats with ovariectomy-induced osteoporosis?
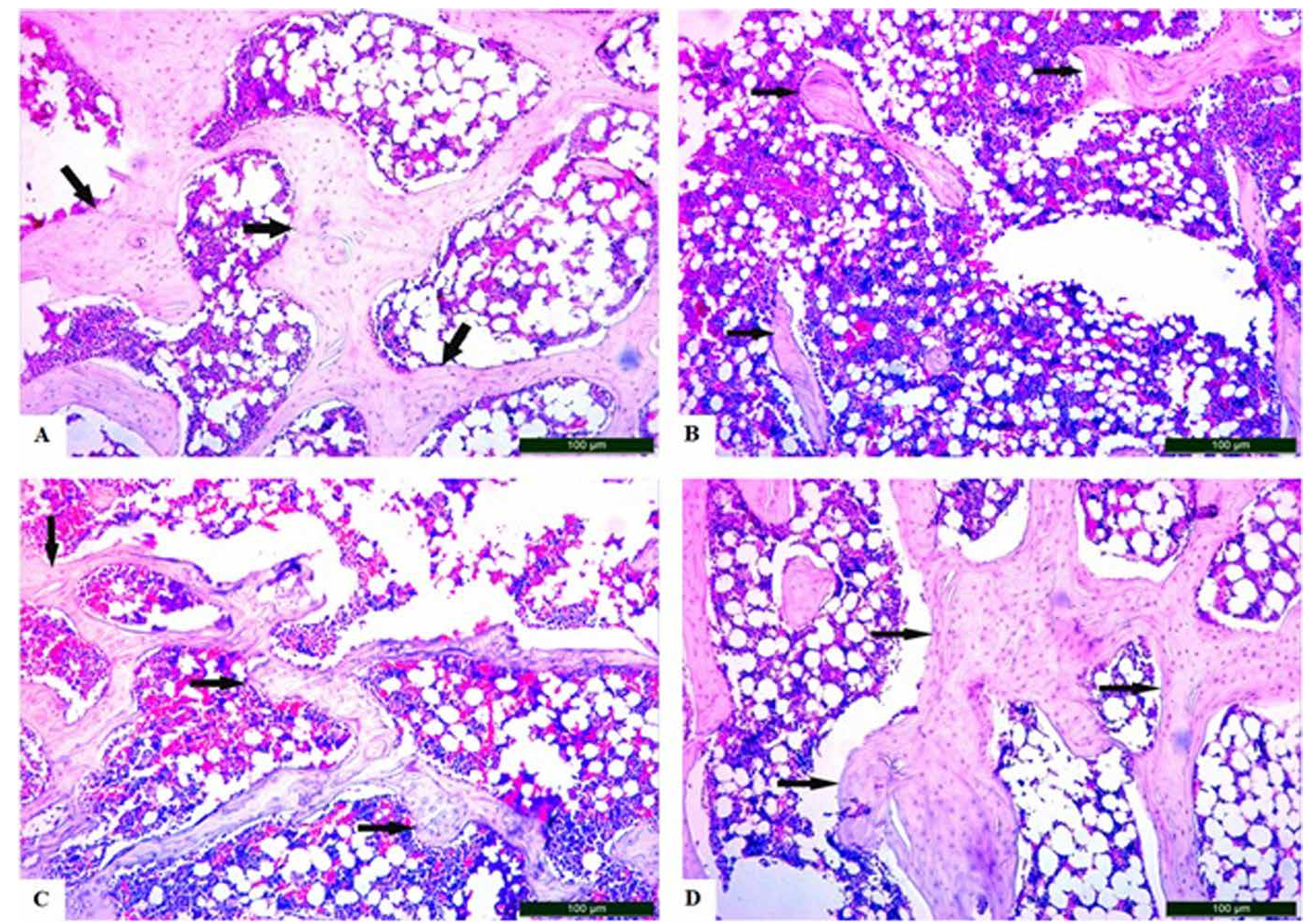

FIGURE 4 - Images showing the right femur epiphyseal bone width in rats with ovariectomy-induced osteoporosis. A: Control group, B: OVX group, C: OVX+zaprinast group and D: OVX+avanafil group. The area or distance between the two arrows indicates the epiphyseal area (bone growth plaque). Scale bar: $50 \mu \mathrm{m}$.

\section{Changes in the Levels of Some Selected Oxidative Stress Parameters}

The CoQ10/CoQ10H and 8-OHdG/106 dG ratios and MDA values were increased to some extent in the OVX group, while they were maintaned at levels similar to the sham group in the OVX+zaprinast and OVX+avanafil groups ( $\mathrm{p}>0.05$, Table I).

Epidemiological studies in humans and mechanical studies in animals showed that oxidative damage is a critical pathogenic factor associated with OP (Manolagas, 2010). Oxidative stress plays a key role in pathophysiological processes, including postmenopausal bone loss and aging (Sendur et al., 2009). In addition, increased oxidative stress and a decreased antioxidant defense capacity in the femurs of the rats with ovariectomy have been reported (Muthusami et al., 2005).

Recent studies of humans and animals have reported that PDE-5 inhibitors (such as sildenafil, vardenafil, tadalafil, avanafil and zaprinast) exert antioxidant effects on different tissues in addition to their angiogenesis effects (Fan et al., 2013). In our literature review, we were unable to find any study on the effects of zaprinast and avanafil on the antioxidant capacity, lipid profile, lipid peroxidation, mitochondria and oxidative DNA damage in human or animal models with OP in vivo and in vitro.

MDA is the end product of lipid peroxidation and co-occurs with other oxidative stress parameters (Huyut et al., 2018). Effendy and Shuid (2014) reported higher MDA levels in ovariectomized rats than in normal rats. Consistent with the studies and findings described above, MDA levels were increased to some extent in the positive control group with ovariectomy compared to the sham group in the present study. Consistent with the studies (Fan et al., 2013) of the antioxidant activity PDE-5Is listed above, we observed that zaprinast and avanafil significantly preserved the MDA levels compared to the ovariectomized positive control group, the levels were similar to the sham group.

Urine and serum $8-\mathrm{OHdG}$ levels are frequently used as a biomarker of oxidative DNA damage in human studies (Qian et al., 2012). Isomura et al (2004) described urinary 8-OHdG levels in postmenopausal rats as a significant indicator of oxidative stress. Qian et al. (2012) reported higher urine 8-OHDG/mg creatinine levels in postmenopausal women with osteopenia at baseline 
and, 1, 3 and 6 months after treatment in the placebo groups than in groups treated with the other drugs. In our literature review, we were unable to find any study investigating the effects of zaprinast and avanafil on 8-OHdG or $8-\mathrm{OHdG} / 10^{6} \mathrm{dG}$ and oxidized CoQ10 levels. In present study, oxidized CoQ10/reduced CoQ10 ratios were increased in the OVX group. In addition, an increase CoQ10/CoQ10H ratios was not observed in the zaprinast and avanafil treated groups with ovariectomy, and the levels were similar to the sham group. The rates of $8-\mathrm{OHdG} / 10^{6} \mathrm{dG}$ ratios in the ovariectomized positive control group were higher than in the sham group, while significantly lower $8-\mathrm{OHdG} / 10^{6} \mathrm{dG}$ ratios were observed in the zaprinast and avanafil treated groups with ovariectomy than in the OVX group (Table I). However, the differences in this parameters between groups were not statistically significant. These data support the previous findings of increased oxidative stress due to acute estrogen deficiency induced by ovariectomy. Morover, zaprinast and avanafil may inhibit the increase in oxidative stress observed in ovariectomized rats.

\section{CONCLUSIONS}

Based on the results of the present study, bone mineral density was decreased and oxidative stress was increased due to acute estrogen deficiency in the female rats with ovariectomy. However, zaprinast and avanafil significantly increased angiogenesis in bone tissue by activating the NO/cGMP/PKG metabolic pathway via cGMP and PKG and reduced progressive BMD loss, bone atrophy and oxidative stress due to acute estrogen deficiency in rats with ovariectomy induced OP. These results provide important clues that PDE-5 inhibitors may prevent increases in bone atrophy, bone loss and oxidative stress in subject with osteoporosis. However, the mechanisms by which zaprinast and avanafil prevent the development of BMD loss, bone atrophy and oxidative stress due to acute estrogen deficiency, are exactly unknown, and new and detailed studies at the molecular level are needed.

\section{ACKNOWLEDGEMENTS}

This study was supported by "Presidency of the Individual Research Projects", Van Yuzuncu Y1 University (2013-TF-B006) and with opportunities from the Department of Biochemistry, Faculty of Medicine, Van Yuzuncu Y1l University and Ataturk University.

\section{DISCLOSURE STATEMENT}

The authors report no conflicts of interest.

\section{ETHICALS APPROVAL}

The study protocol was performed in accordance with the Declaration of Helsinki as revised in 2000. The study protocol was approved by the local ethics committee, and informed consent was obtained from each subject.

\section{REFERENCES}

Alp HH, Huyut Z, Yildirim S, Başbugan Y, Ediz L, Şekeroğlu MR. The effect of PDE5 inhibitors on bone and oxidative damage in ovariectomy induced osteoporosis. Exp Biol Med. 2017;242(10):1051-1061.

Anderson KE. Pharmacology of penile erection. Pharmacol Rev. 2001;53(3):417-450.

Armour KA, Armour KJ, Gallagher ME, Decke AG, Helfrich MH, Reid DM et al. Defective bone formation and anabolic response to exogenous estrogen in mice with targeted disruption of endothelial nitric oxide synthase. Endocrinology. 2001;142(2):760-766.

Chow SK, Leung K, Qin L, Wei F, Cheung W. Callus formation is related to the expression ratios of estrogen receptors alpha and beta in ovariectmy induced osteoporetic fracture healing. Arch Orthop Trauma Surg. 2014;134(10):1405-1416.

Effendy NM and Shuid AN. Time and dose dependent effects of labisia pumila on bone oxidative status of postmenopausal osteoporosis rat model. Nutrients. 2014;6(8):288-3302.

El-Nabarawi N, El-Wakd M, Salem M. Atorvastatin, a double weapon in osteoporosis treatment: an experimental and clinical study. Drug Des Dev Ther. 2017;11:1383-1391.

Fan X, Roy E, Zhu L, Murphy TC, Ackert-Bicknell C, Hart $\mathrm{CM}$ et al. Nitric oxide regulates receptor activator of nuclear factor-kappa B ligand and osteoprotegerin expression in bone marrow stromal cells. Endocrinology. 2004;145(2):751-759.

Fan YF, Zhang R, Jiang X, Wen L, Wu DC, Liu D et al. The phosphodiesterase-5 inhibitor vardenafil reduces oxidative stress while reversing pulmonary arterial hypertension. Cardiovasc Res. 2013;99(3):395-403.

Firat SC, Cetin Z, Samanci N, Aydin F, Balci N, Gungor M et al. Evaluation of eNOS gene polymorphisms in relation to BMD in postmenopausal women. Maturitas. 2009;63(4): 352-356. 
Do avanafil and zaprinast exert positive effects on bone tissue via the nitric oxide/cyclic guanosine monophosphate/protein kinase-G signaling pathway in rats with ovariectomy-induced osteoporosis?

Huyut Z, Bakan N, Yildirım S, Alp HH. Effects of the Phosphodiesterase-5 (PDE-5) inhibitors, avanafil and zaprinast, on bone remodeling and oxidative damage in a rat model of glucocorticoid-induced osteoporosis. Med Sci Mon Bas Res. 2018;24:47-58.

Isomura H, Fujie K, Shibata K, Inoue N, Iizuka T, Takebe G et al. Bone metabolism and oxidative stress in postmenopausal rats with iron overload. Toxicology. 2004;197(2):92-99.

Kaleta B, Boguska A. Sildenafil, a phosphodiesterase type 5 inhibitor, downregulates osteopontin in human peripheral blood mononuclear cells. Arc Immunol Ther Exp. 2017;65(4):347-353.

Kanis JA, Johnell O. Ten year risk of osteoporotik fracture and the effect of risk factors on screening strategies. Bone. 2002;30(1):251-258.

Kass DA, Takimoto E, Nagayama T, Chamption HC. Phosphodiesterase regulation of nitric oxide signalling. Cardiovasc Res. 2007;75(2):303-314.

Kaur $\mathrm{H}$ and Halliwell B. Measurement of oxidized and methylated DNA bases by HPLC with electrochemical detection. Biochem J. 1996;318(1):21-23.

Khoschsorur GA, Winklhofer-Roob BM, Rabl H, Auer T, Peng Z, Schaur RJ. Evaluation of a sensitive HPLC method for the determination of malondialdehyde, and application of the method to different biological materials. Chromatographia. 2000;52(3-4):181-184.

Kim SW, Jeong S, Munarriz R, Kim NN, Goldstein I, Traish AM. An in vivo rat model to investigate female vaginal arousal response. J Urology. 2004;171(3):1357-1361.

Li F, Sun X, Ma J, Ma X, Zhao B, Zhang Y et al. Naringin prevents ovariectomy induced osteoporosis and promotes osteoclasts apoptosis through the mitochondria mediated apoptosis pathway. Biochem Bioph Res Co. 2014;452(3):629635.

Lien TF, Hsu YL, Lo DY and Chiou RYY. Supplementary health benefits of soy aglycons of isoflavone by improvement of serum biochemical attributes, enhancement of liver antioxidative capacities and protection of vaginal epithelium of ovariectomized rats. Nutr Metab. 2009;6:15.

Littarru GP, Mosca F, Fattorini D, Bompadre S, Battino M. Assay of coenzyme Q10 in plasma by a single dilution step. Meth Enzymol. 2004;378:170-176.

Mada SB, Reddi S, Kumar N, Kumar R, Kapila S, Kapila R et al. Antioxidative peptide from milk exhibits antiosteopenic effects through inhibition of oxidative damage and bone- resorbing cytokines in ovariectomized rats. Nutrition. 2017;43-44:21-31.

Mancini L, Moradi-Bidhendi N, Becherini V, Martineti I, Mclntyre I. The biphasic effects of nitric oxide in primary rat osteoblasts are cGMP dependent. Biochem Bioph Res Co. 2000;274(2):477-481.

Manolagas SC. From estrogen-centric to aging and oxidative stress: a revised perspective of the pathogenesis of osteoporosis. Endocr Rev. 2010;31(3):266-300.

Martineau P, Leslie WD. Trabecular bone score (TBS): Method and applications. Bone. 2017;104:66-72.

Miettinen M, Lindenmayer AE, Chanbal A. Endothelial cell markers CD31, CD34, and BNH9 antibody to $\mathrm{H}$ - and $\mathrm{Y}$ - antigens: evaluation of their specificity and sensitivity in the diagnosis of vascular tumors and comparison with von Wi1lebrand factor. Modern Pathol. 1994;7:82-90.

Muthusami S, Ramachandran I, Muthusamy B, Vasudevan G, Prabhu V, Subramaniam V et al. Ovariectomy induces oxidative stress and impairs bone antioxidant system in adult rats. Clin Chim Acta. 2005;360:81-86.

Rahal A, Kumar A, Singh V, Yadav B, Tiwari R, Chakraborty $\mathrm{S}$ et al. Oxidative stress, prooxidants, and antioxidants: The interplay. BioMed Res Int. 2014;2014:1-19.

Sendur OF, Turan Y, Tastaban E, Serter M. Antioxidant status in patients with osteoporosis: A controlled study. Joint Bone Spine 2009;76:514-518.

Sharad D, Parth B, Madhavi N, Kunal S, Archana P. Antiosteoporotic effect of Hemidesmus indicus Linn on ovariectomised rats. J Ethnopharmacol. 2017;199:1-8.

Shi SR, Gu J, Krishan LK, Taiying C, Richard JC, Taylor CR. A novel approach to immunohistochemistry on routinely processed tissue sections", in: Gu J (ed.) Analitical morphology theory, applications and protocols. 1st ed. Basel: Birkhauser, 1996; pp. 1-40.

Yaman F, Atılgan S, Günes N, Agacayak S, Gunay A, Ucan MC et al. Phosphodiesterase-5 inhibitors may facilitate bone defect recovery. Eur Rev Med Pharmacol. 2011;15(11):1301-1305.

Zheng S, Qin S, Zhong ZM, Wu Q, Ding RT, Liao CR et al. Advanced oxidation protein products (AOPPs) accelerate bone loss in rats. Int J Clin Exp Pathol. 2016;9(11):11289-11300.

Zhang DW, Deng H, Qi W, Zhao G,Cao W. Osteoprotective effect of cordycepin on estrogen deficiency induced osteoporosis in vitro and in vivo. BioMed Res Int. 2015;2015:423869. 
Zhou Q, Zhu L, Zhang D, Li N, Li Q, Dai P et al. Oxidative stress related biomarkers in postmenopausal osteoporosis: a systematic review and meta-analyses. BioMed Res Int. 2016:1-12.

Zhou H, Xie Y, Baloch Z, Shi Q, Huo Q, Ma T. The effect of atorvastatin, 3-hydroxy-3-methylglutaryl coenzyme A reductase inhibitor ( $\mathrm{HMGCoA}$ ), on the prevention of osteoporosis in ovariectomized rabbits. J Bone Miner Metab. 2017;35(3):245-254.

Xing LZ, Ni HJ, Wang YL. Quercitrin attenuates osteoporosis in ovariectomized rats by regulating mitogen-activated protein kinase (MAPK) signaling pathways. Biomed Pharmacother. 2017;89:1136-1141.

Qian G, Xue K, Tang L, Wang F, Song X, Chyu MC et al. Mitigation of oxidative damage by green tea polyphenols and Tai Chi Exercise in postmenopausal women with osteopenia. PLOS ONE 2012;7:e48090.
Wie J, Jeong SJ, Kwak M, Myeong J, Chae M, Park JK et al. The regulation of transient receptor potential canonical 4 (TRPC4) channel by phosphodiesterase 5 inhibitor via the cyclic guanosine 3',5'-monophosphate. Pflug Arch Eur J Phy. 2017;469(5-6):693-702.

Wimalawansa SM, Shankar VS, Simmins DJ, Wimalawansa SJ. The mechanism of bone resorption by cyclosporin: involvement of the NO-cGMP pathway. J Musculoskel Neuron. 2000;1(2):141-143.

Wu Y, Cao LY, Xia L, Wu Q, Wang J, Wang X et al. Evaluation of osteogenesis and angiogenesis of icarin in local controlled release and systemic delivery for calvarial defect in ovariectomized rats. Sci Rep. 2017;7(1):1-14.

Received for publication on $15^{\text {th }}$ November 2018 Accepted for publication on 01 ${ }^{\text {st }}$ April 2019 